\title{
The liver transcriptome of two full-sibling Songliao black pigs with extreme differences in backfat thickness
}

\author{
Kai Xing ${ }^{1}$, Feng Zhu' ${ }^{1}$ Liwei Zhai ${ }^{1}$, Huijie Liu' ${ }^{1}$ Zhijun Wang ${ }^{2}$, Zhuocheng Hou ${ }^{1 *}$ and Chuduan Wang ${ }^{1 *}$
}

\begin{abstract}
Background: Fatness traits in animals are important for their growth, meat quality, reproductive performance, and immunity. The liver is the principal organ of the regulation of lipid metabolism, and this study used massive parallelized high-throughput sequencing technologies to determine the porcine liver tissue transcriptome architecture of two full-sibling Songliao black pigs harboring extremely different phenotypes of backfat thickness.

Results: The total number of reads produced for each sample was in the region of 53 million, and 8,226 novel transcripts were detected. Approximately 92 genes were differentially regulated in the liver tissue, while 31 spliced transcripts and 33 primary transcripts showed significantly differential expression between pigs with higher and lower backfat thickness. Genes that were differentially expressed were involved in the metabolism of various substances, small molecule biochemistry, and molecular transport.
\end{abstract}

Conclusions: Genes involved in the regulation of lipids could play an important role in lipid and fatty acid metabolism in the liver. These results could help us understand how liver metabolism affects the backfat thickness of pigs.

Keywords: Backfat thickness, Liver, Pig, RNA-Seq

\section{Background}

Consumer choice of pig meat is an important factor that dictates the principles of swine breeding worldwide. One aspect of this is the deposition of fat in the muscle and backfat, which is associated with growth rate, meat quality, and reproductive performance [1]. Backfat thickness is highly correlated with body fat radio, carcass crosssectional fat area ratios, and intramuscular fat, so is a good indicator of fat deposition in pigs [2]. Therefore, the selection of backfat thickness using B-mode real-time ultrasound is a practical and economical method in pig breeding for increasing feeding efficiency, carcass value, and consumer acceptance of pork [3]. Because of the increasing problem of human obesity worldwide, it is beneficial to regulate fat deposition in pig breeding through molecular markers. Additionally, their similarity to humans in body size and other physiological/anatomical features, including their

\footnotetext{
* Correspondence: zchou@cau.edu.cn; cdwang@cau.edu.cn

${ }^{1}$ National Engineering Laboratory for Animal Breeding and MOA Key Laboratory of Animal Genetics and Breeding, Department of Animal Genetics and Breeding, China Agricultural University, 100193 Beijing, China Full list of author information is available at the end of the article
}

innate tendency to over consume food, means that pigs are a good animal model for studying obesity [4].

The extent of fat deposition can be determined by triacylglycerol synthesis and storage, lipid mobilization, and fatty acid oxidation [5]. The liver is one of the most important organs to regulate appetite and body weight in pigs, as well as playing a key role in regulating several metabolic processes [6]. In pigs, de novo cholesterol synthesis and fatty acid oxidation mainly take place in the liver [7]. Lipid hydrolysis in adipose tissue results in free fatty acid, which is combined with plasma albumin then transported to the liver for use as an energy source through oxidation [8].

RNA-Seq technology for transcriptome profiling has previously been used to explore the transcriptome of pig liver tissue. Such transcriptomes were recently compared between a full-sibling (full-sib) pair of F2 females from a White Duroc $\times$ Erhualian resource population with extreme phenotypes in growth and fat deposition [9]. Other comparisons include a backcross of two female groups $(\mathrm{H}$ and $\mathrm{L})$ with extreme intramuscular fatty acid composition (25\% Iberian $\times 75 \%$ Landrace) [10], and Duroc $\times$ F2 (Leicoma $\times$ German Landrace) cross 
pigs with divergent skatole levels in backfat [11]. Although several studies have previously attempted to identify the genes and pathways involved in fatty traits in the liver, to our knowledge, they either lacked sufficient RNA-Seq liver samples or did not take into account the effect of different genetic background noise when analyzing liver regulation.

The Songliao black pig is a Chinese domestic breed with powerful stress resistance and good reproductive and fat deposition capabilities. It is therefore a good model for studying fatty deposition. In the present study, we used RNA-Seq to obtain the liver transcriptomes of two full-sib Songliao black pigs with a high variation in backfat thickness. The main aim of this study was to elucidate the genes and pathways involved in lipid metabolism in liver tissue using RNA deep sequencing technology.

\section{Methods}

\section{Experimental design, animals, and phenotypes}

The Songliao black female pig population (average age, 217 (range, 216-218) days; average live weight, $100 \mathrm{~kg}$ (range, 92.5-116.4 kg) was housed in consistent and standard environmental conditions with natural, uncontrolled room temperature and light. Animals were fed three times a day and had access to water ad libitum. Pedigree information was available for all animals. Live backfat thickness was measured on the last 3/4 rib using B-mode real-time ultrasound (HS1500, Honda, Japan). We analyzed a total of 53 individuals with full/half-sibs for backfat thickness to identify pairs with two divergent phenotypes. To minimize the noise of different genetic back grounds, full-sibs were selected as a priority.

We set out to compare transcriptome changes between two groups with a high variation in backfat thickness: pigs with higher backfat thickness (BH) and those with lower backfat thickness (BL) which had a backfat thickness 2-3 times lower than that of $\mathrm{BH}$ pigs. The chosen animals also had to have a similar backfat thickness within the same group (BH/BL) after adjustment for live body weight. Based on our criteria, experimental samples were made up of two pairs of pigs with extreme backfat thickness differences, both of which were full-sibs.

The chosen pigs were slaughtered according to guidelines for the ethical use and treatment of animals in experiments in China. Liver tissue was separated and stored in liquid nitrogen until analyzed. Total liver RNA was extracted using the total RNA extraction Kit (Bioteke, China) according to the manufacturer's recommendations. The quality of total RNA was assessed by the 2100 Bioanalyzer (Agilent, USA).

\section{mRNA library construction and sequencing}

mRNA was isolated from total RNA samples using oligo (DT) magnetic beads (Invitrogen, USA). Purified mRNA was first fragmented by the RNA fragmentation kit (Ambion, USA), then a one paired-end library was prepared for each sample according to the manufacturer's instructions. mRNA libraries were individually sequenced for foursamples (two from each of the $\mathrm{BH}$ and BL group) using the Illumina High-seq 2000 sequencing system. The libraries were sequenced using a multiplexed paired-ends protocol with $180 \mathrm{bp}$ of data collected per run. The average insert size for the paired-end libraries was $180 \mathrm{bp}$. A total of four paired-end mRNA libraries were constructed individually for four liver samples.

\section{Mapping and counting reads}

Quality control and reads statistics were determined using FASTQC (http://www.bioinformatics.babraham.ac.uk/projects/fastqc/). All reads were trimmed 20-bp from the 5' end according to the reads quality distributions. After removal of the sequencing adapt and low-complexity reads, all RNA-Seq reads were mapped on the reference pig genome (Sscrofa10.2) using TopHat v2.0.1 software [12] with default parameters. The annotation database Ensembl Genes v67 was used as a reference. Additionally, the intersect from BED Tools was used to count the number of reads mapping to exons, introns, and intergenic positions in the genome [13]. The reads count was measured using easy RNASeq software [14] to quantify the raw reads mapped on each gene.

\section{Differential expression and novel transcript analysis}

The trimmed mean of M-values (TMM) was used to normalize gene expression levels [15]. After normalization, the NOISeq package implanted in the R computation environment was used to detect differentially expressed genes (DEGs) between two groups [16]. This method infers the noise distribution from the data and performs pair wise comparisons of the samples to identify DEGs. To measure expression level changes between two conditions, NOISeq takes into consideration two statistics: $\mathrm{M}$ (the $\log _{2}$-ratio of the two conditions) and $\mathrm{D}$ (the absolute value of the difference between conditions). The probability thresholds were $P \geq 0.8$ and the TMM value in the lower expressed sample was $\geq 1$. The higher the probability, the greater the change in expression between the two groups. Using a probability threshold of 0.8 means that the gene is 4 times more likely to be differentially expressed than non-differentially expressed

\begin{tabular}{|c|c|c|c|c|}
\hline ID & $\mathrm{H} 710$ & $\mathrm{H} 712$ & H906 & H909 \\
\hline Backfat thickness of live, $\mathrm{mm}$ & 24.9 & 9.4 & 21.7 & 8.8 \\
\hline Backfat thickness of carcass, $\mathrm{mm}$ & 37.0 & 18.9 & 31.7 & 15.5 \\
\hline Kidney fat, kg & 1.45 & 0.7 & 1.5 & 0.75 \\
\hline
\end{tabular}

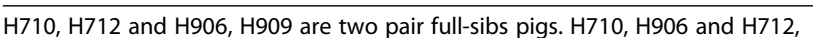
$\mathrm{H} 909$ are the BH and BL groups respectively. 
Table 2 The number of reads obtained and percentages of mapped reads per sample

\begin{tabular}{|c|c|c|c|c|c|c|}
\hline Sample ID ${ }^{1}$ & Total reads number, $\mathrm{M}^{2}$ & Mapping rate, $\%^{3}$ & CDS exons, \% & 5'UTR, \% & 3'UTR, \% & Intron, \% \\
\hline $\mathrm{H} 906$ & 53.08 & 84.80 & 72.85 & 1.78 & 10.43 & 6.45 \\
\hline $\mathrm{H} 909$ & 52.16 & 92.90 & 76.21 & 2.64 & 7.98 & 5.11 \\
\hline $\mathrm{H} 710$ & 54.88 & 87.86 & 75.85 & 1.81 & 9.77 & 5.01 \\
\hline $\mathrm{H} 712$ & 51.56 & 88.17 & 75.28 & 1.79 & 8.89 & 6.35 \\
\hline
\end{tabular}

1. $\mathrm{H} 710, \mathrm{H} 712$ and $\mathrm{H} 906, \mathrm{H} 909$ are two pair full-sibs pigs. $\mathrm{H710}, \mathrm{H} 906$ and $\mathrm{H} 712, \mathrm{H} 909$ are the $\mathrm{BH}$ and $\mathrm{BL}$ groups respectively.

2. Indicate millions of reads.

3. Use the Ensembl V68 as the reference genome annotation to classify the mapping tags into the different regions. Ratio of the tags mapping on the subregion of the gene was calculated as the tags on each region divided by the total tags on the whole genome.

[17]. Novel transcripts, differentially expressed spliced transcripts, and primary transcripts were also detected using the Cufflinks suite of software for RNA-Seq [12].

\section{Functional enrichment analysis of differentially expressed genes}

Because the pig genome is poorly annotated, pig gene IDs were converted to human gene IDs using BioMart. DEG lists were submitted to the Database for Annotation, Visualization and Integrated Discovery (DAVID) bioinformatics resource for enrichment analysis of the significant overrepresentation of GO biological processes (GO-BP), molecular function (GO-MF), cellular component (GOCC), and KEGG-pathway category [18]. In all tests, $P$-values were calculated using Benjamini-corrected modified Fisher's exact test and $\leq 0.05$ was taken as a threshold of significance.

To further identify the DEG interaction network in the liver, the Search Tool for the Retrieval of Interacting Genes (STRING) was used, which is based on a known proteinprotein interaction database program. It generates a network of interactions from a variety of sources, including different interaction databases, text mining, genetic interactions, and shared pathway interactions [19].

\section{Quantitative PCR and data analysis}

Total RNA was extracted from the liver and converted into cDNA using the Revert Aid ${ }^{\mathrm{TM}}$ First Strand cDNA Synthesis Kit (Thermo Fisher Scientific Inc, USA) following the manufacturer's protocol. cDNA samples were analyzed with real-time reverse transcriptase (RT)-PCR using the Light Cycler 480 Real-Time PCR System (Roche, USA). RT-PCR reactions were performed in a final volume of 20 $\mu \mathrm{l}$ with the Roche SYBR Green PCR Kit (Roche) according to the manufacturer's instructions. Pig GAPDH was used as an internal standard to correct the cDNA input. Triplicate RT-qPCRs were performed for each cDNA and the average $\mathrm{Ct}$ was used for further analysis. Relative quantification values were calculated using the $2^{-\Delta \Delta C t}$ method.

\section{Data availability}

Complete data sets have been submitted to NCBI Sequence Read Archive (SRA) under Accession no. SRP035376, Bioproject: PRJNA234465.

\section{Results}

\section{Analysis of RNA deep sequencing data}

The backfat thickness of the pig carcass and weight of the kidney were shown to differ greatly between groups (Table 1). In general, individuals in the $\mathrm{BH}$ group had twice the backfat thickness compared with those in the BL group. Our experimental population is a conserved breed that has not undergone extensive selection nor hybridized with other breeds. Individuals therefore have very similar genetic backgrounds.

We obtained approximately 53 million paired-end clean reads of $90 \mathrm{bp}$ for each sample, and high percentages of mapped reads ranging from 84.80 to $92.90 \%$. The number of reads and percentages of mapped reads were similar between the two groups (Table 2). Most mapped reads were located within an exon, with percent ages ranging from 72.85 to $76.21 \%$. Other reads mapped within the untranslated region, introns, and intergenic regions. The percentages of reads in each region are shown in Table 2.

The total number of genes expressed in the liver in the four samples ranged from 16,815 to 17,025 (Additional file 1: Table S1), with numbers of expressed genes being similar between the two groups. Correlations between biological replicate samples showed that the expressed genes were very highly reproducible, suggesting that a major fraction of the liver transcriptome is conserved between groups.

To confirm changes in transcript levels between $\mathrm{BH}$ and BL groups, six genes related to fatty acid synthesis or lipid metabolism were selected for RT-PCR analysis: ACACA, LDHA, ELOVL6, CYP1A2, PDK1, and SCD. We designed RT-PCR primers (Additional file 2: Table S2) for these genes, using GAPDH as a reference. When gene expression levels were compared, a strong correlation between RT-qPCR and RNA-Seq platforms was observed (0.72), confirming the high reproducibility of the data. For all six genes, the fold-change ratios between $\mathrm{H}$ and $\mathrm{L}$ groups were consistent with the RNASeq data (Figure 1).

\section{Differentially expressed genes between $\mathrm{H}$ and $\mathrm{L}$ groups} We quantified transcript expression levels in TMM to normalize gene expression data across different samples. 


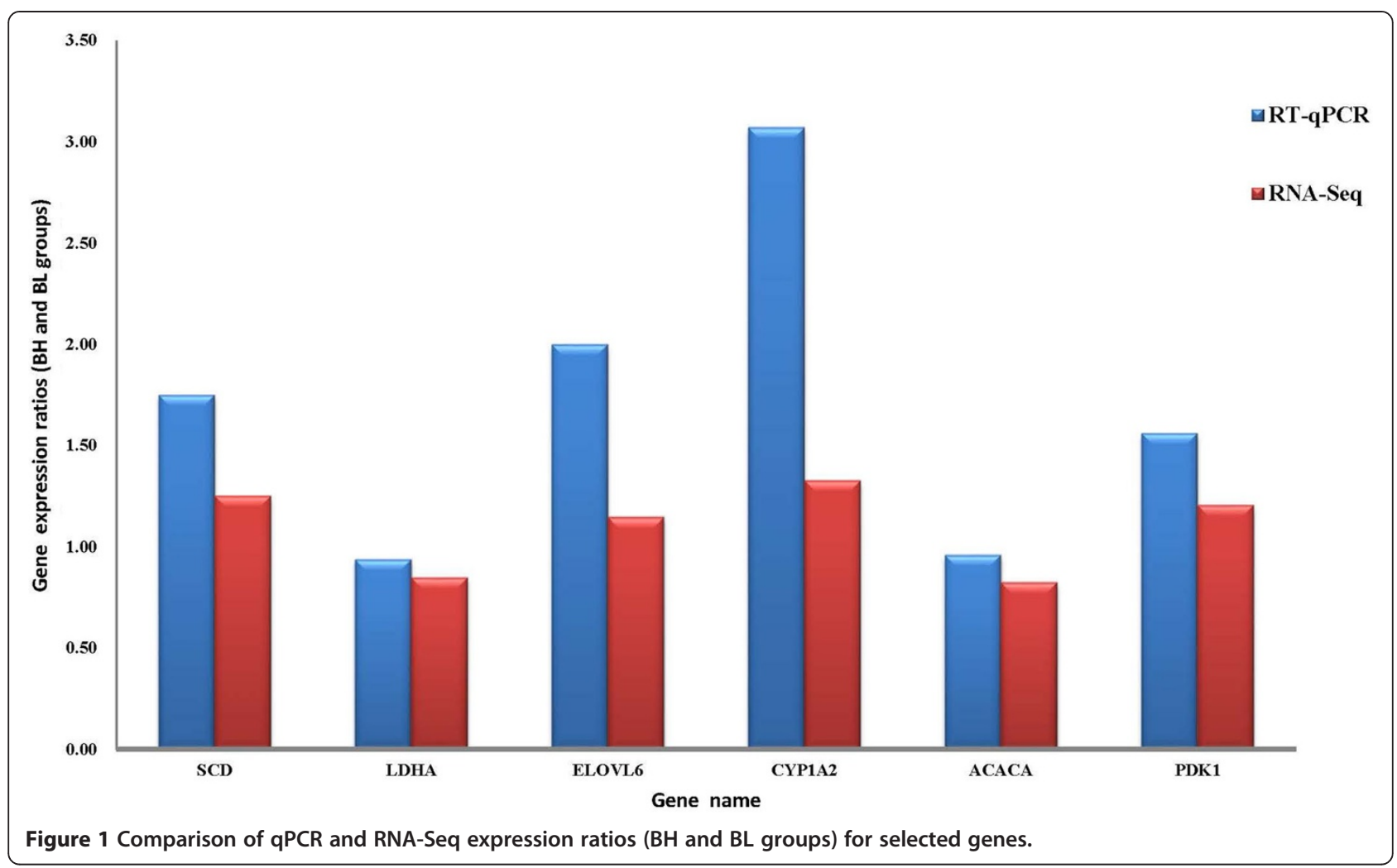

Differential gene expression in liver tissue was calculated from the raw reads using the NOISeq package. We treated high/low backfat thickness samples as biological replicates because they showed similar phenotypes (Table 1). This process identified 92 liver DEGs between pigs with extreme high and low backfat thickness levels (Additional file 3: Table S3) We also identified 587 and 690 genes that were only expressed in the BH or BL group, respectively (Figure 2). Comparing shared DEGs between the two different pairs of pigs, we found that DEGs of biological replicates were more homogeneous with fewer false positives. Because of the sample limitations for each pair, we only

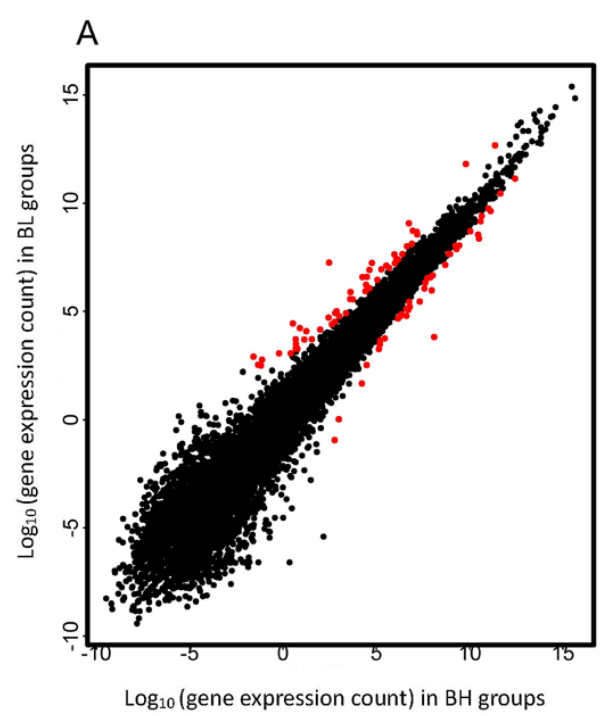

B

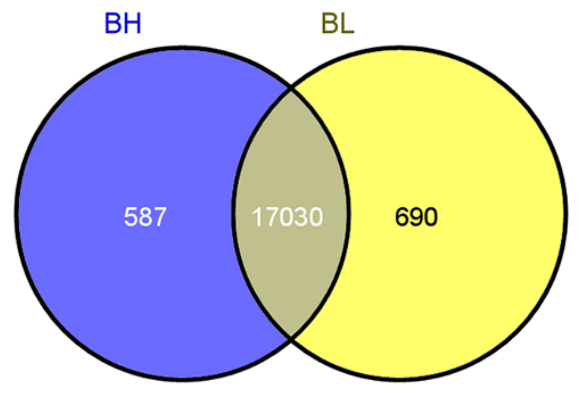

Figure 2 Gene expression in BH and BL groups. A. Red points represent genes that are significantly differentially expressed. B. Venn diagram showing genes only expressed in the BH group (blue circle), only expressed in the BL group (yellow circle), and common to both groups (intersection). 
Table 3 Pathways enriched in up-regulated genes in the liver (BH vs. BL)

\begin{tabular}{|c|c|c|c|}
\hline Pathway & Gene & $P$ value & Benjamini \\
\hline Metabolism of xenobiotics by cytochrome P450 & $\begin{array}{l}\text { CYP1A2, LOC100515394,LOC100526118, CYP1A1, LOC100511647, } \\
\text { ADH1A CYP2S1 }\end{array}$ & $1.53 \mathrm{E}-08$ & 4.43E-07 \\
\hline Drug metabolism & CYP1A2, LOC100515394, GSTM4 LOC100511647, ADH1A, CYP2A6 & $9.02 \mathrm{E}-07$ & $1.31 \mathrm{E}-05$ \\
\hline Retinol metabolism & CYP1A2, LOC100515394, CYP1A1 ADH1A, CYP2A6 & $1.88 \mathrm{E}-05$ & $1.82 \mathrm{E}-04$ \\
\hline Tryptophan metabolism & CYP1A2, CYP1A1, OGDHL & 0.006753 & 0.047941 \\
\hline
\end{tabular}

present functional analysis of DEGs obtained by treating the two pig pairs as two biological replicates.

\section{Novel transcripts}

We used the reference annotation based transcript algorithm implemented in Cufflinks to identify novel transcripts that were not annotated in the current Ensembl pig gene annotation database. A total of 8,226 novel transcripts were detected for FPKM $\geq 1$ in the Songliao black pigs. Thirtyone spliced transcripts and 33 primary transcripts were significantly differentially expressed between the two groups (Additional file 4: Table S4).

\section{Functional enrichment analysis of the DEGs}

Of the 92 DEGs, 39 were up-regulated and 53 were down-regulated in the $\mathrm{BH}$ group compared with the $\mathrm{BL}$ group. Pig gene IDs were converted to human gene IDs, but five genes did not match with their human homologs (ENSSSCG00000008012, ENSSSCG00000016695, ENSSSCG00000030368, ENSSSCG00000010427, and ENSSSCG00000012881).

To gain an insight into the liver tissue processes that differ between the $\mathrm{BL}$ and $\mathrm{BH}$ groups of pigs, differentially up-regulated and down-regulated genes underwent separate pathway analysis and gene ontology analysis using DAVID. Human homologs were recognized for 32 of the 39 up-regulated genes, and up-regulated genes were found to be involved in the metabolism of xenobiotics, drugs, retinol, and tryptophan (Table 3). The expression of DEGs in the enriched pathways is shown in Table 4. Following GO analysis, the DEGs were shown to be related to biological processes such as amino acid biosynthesis and

Table 4 Expression levels of genes in up-regulated pathways in the liver (BH vs. BL)

\begin{tabular}{|c|c|c|c|c|}
\hline Pathway & Gene symbol & $\mathrm{BH}$ & $\mathrm{BL}$ & Probability \\
\hline \multirow[t]{7}{*}{ Metabolism of xenobiotics by cytochrome P450 } & CYP1A2 & 260.26 & 100.93 & 0.83 \\
\hline & LOC100515394 & 71.57 & 25.53 & 0.83 \\
\hline & LOC100526118 & 1539.43 & 574.36 & 0.85 \\
\hline & CYP1A1 & 81.60 & 29.08 & 0.83 \\
\hline & LOC100511647 & 3211.75 & 1402.03 & 0.81 \\
\hline & $\mathrm{ADH} 1 \mathrm{~A}$ & 2055.82 & 869.66 & 0.82 \\
\hline & CYP2A6 & 1596.90 & 683.59 & 0.82 \\
\hline \multirow[t]{6}{*}{ Drug metism } & CYP1A2 & 260.26 & 100.93 & 0.83 \\
\hline & LOC100515394 & 71.57 & 25.53 & 0.83 \\
\hline & GSTM4 & 192.04 & 66.50 & 0.85 \\
\hline & LOC100511647 & 3211.75 & 1402.03 & 0.81 \\
\hline & $\mathrm{ADH} 1 \mathrm{~A}$ & 2055.82 & 869.66 & 0.82 \\
\hline & CYP2A6 & 1596.90 & 683.59 & 0.82 \\
\hline \multirow[t]{5}{*}{ Retinol metabolism } & CYP1A2 & 260.26 & 100.93 & 0.83 \\
\hline & LOC100515394 & 71.57 & 25.53 & 0.83 \\
\hline & CYP1A1 & 81.60 & 29.08 & 0.83 \\
\hline & $\mathrm{ADH} 1 \mathrm{~A}$ & 2055.82 & 869.66 & 0.82 \\
\hline & CYP2A6 & 1596.90 & 683.59 & 0.82 \\
\hline \multirow[t]{3}{*}{ Tryptophan metabolism } & CYP1A2 & 81.60 & 29.08 & 0.83 \\
\hline & CYP1A1 & 81.60 & 29.08 & 0.83 \\
\hline & OGDHL & 6.83 & 0.52 & 0.82 \\
\hline
\end{tabular}


metabolism, small molecule metabolism, and oxidation reduction. Forty-five of the 53 down-regulated genes had annotations in DAVID. No significant pathway was found to be associated with down-regulated genes. GO analysis showed that the biological processes enriched by DEGs are complex and relate to protein transport and enzyme activity (Figure 3 ).

\section{Protein-protein interaction analysis}

To gain a better understanding of the biological relationships between genes, the integral DEG list was inputted into the STRING database. Most proteins encoded by DEGs were dissociative, and two protein-protein interaction networks were identified (Figure 4). In one network, genes were associated with metabolism, detoxification, and superoxides, while genes in the other network were related to the heat stress response.

\section{Discussion}

In the present study, the percentages of mapped reads obtained per individual animal (84.80-92.90\%) were higher than those in previous porcine liver transcriptome studies:
61.4-65.6\% [9], 71.42-77.75\% [10], and 43-84\% [11]. Additionally, the percentage of reads within exons was higher than in previous studies, indicating that our results are more effective and credible.

Few studies have investigated how the liver transcriptome affects fat deposition. Our current gene expression analyses show that DEGs and pathways may play very important roles in this, and that genes involved in material metabolism and fatty acid transport are more active in the fatter groups of animals. These findings are consistent with those of previous studies. Previously, the expression levels of CA3, SERPINA6, GATM, GSTA2, and ALAS1 were also found to be significantly different between the two groups [9]. $C A 3$ is associated with the internal fat rate and backfat thickness ofthe pig [20], while SERPINA6 within the quantitative trait loci is associated with cortisol levels, fat, and muscle content, so can be considered a key regulator of obesity susceptibility [21]. These findings indicate that CA3 and SERPINA6 are strongly associated with fatty traits.

It is noteworthy that several DEGs identified in the present study (FABP-1, LCN2, PLIN2, CYP1A1, CYP1A2,

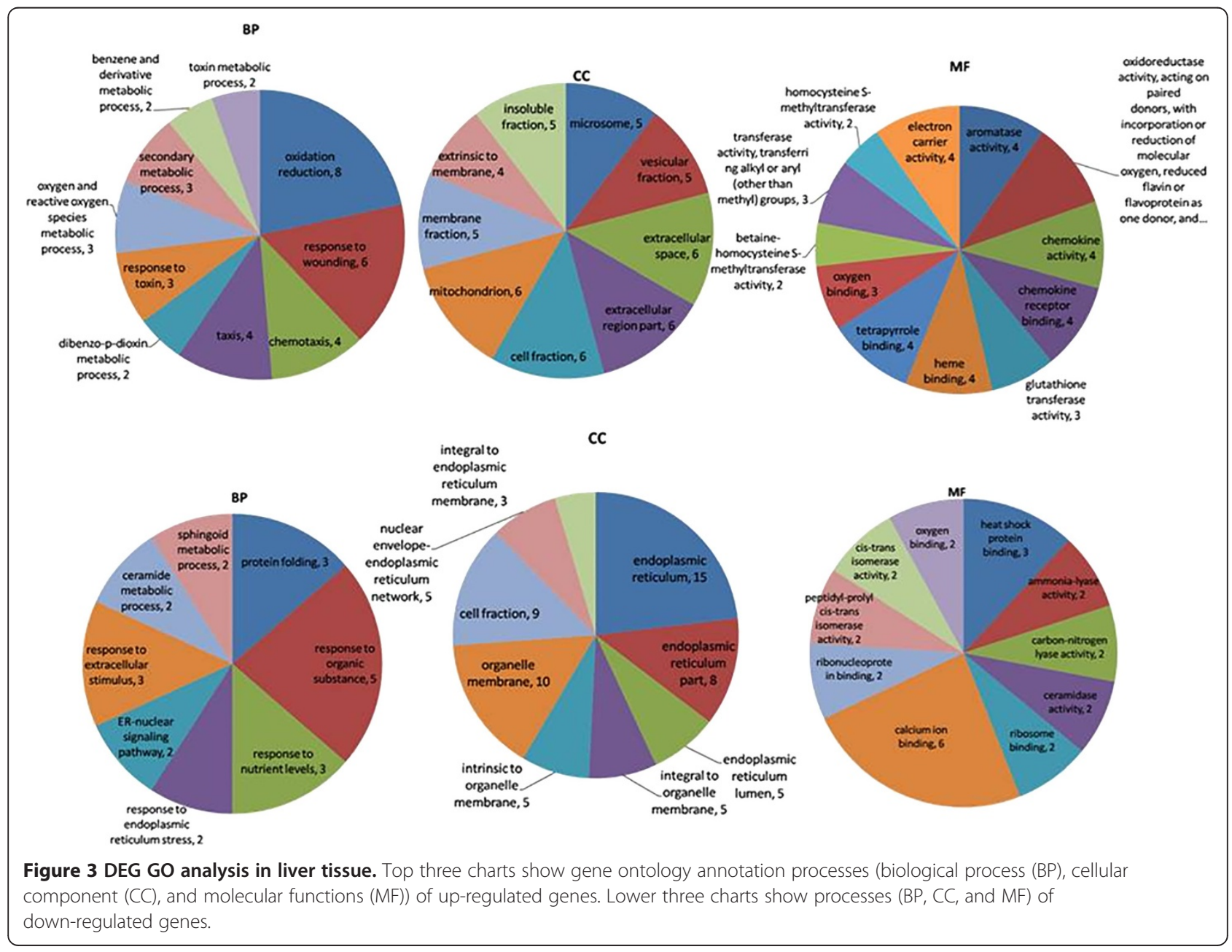




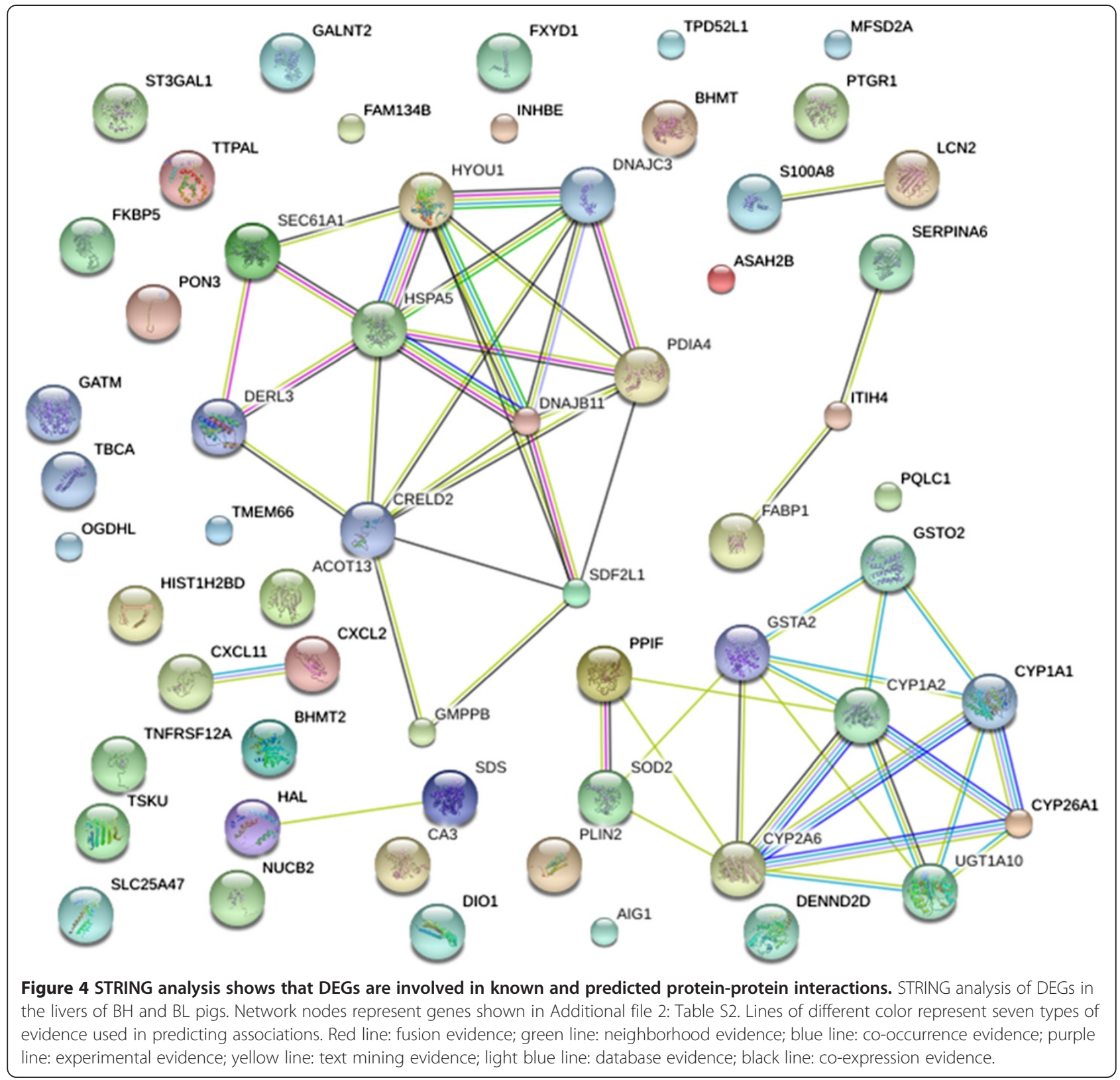

CYP2A6, and CYP26A1) are involved in lipid metabolism. As a member of the family of fatty acid-binding proteins, $F A B P-1$ was expressed in all 12 tissues studied in a previous investigation, but transcript levels were more abundant in the liver and small intestine [22]. FABP-1 is involved in the transport of fatty acids to the site of $\beta$-oxidation, as well as the synthesis of triacylglycerol and phospholipids. It is also shown to interact with peroxisome proliferator-activated receptor (PPAR) $\alpha$, which regulates fatty acid catabolism [23]. Compared with leaner pigs, FABP mRNA and protein levels were expressed at higher levels in the muscle of fatter animals [24]. In the present study, FABP-1 was up-regulated in the liver samples of $\mathrm{BH}$ pigs compared with BL. Consistent with our results, FABP-1 was also up-regulated in obese ob/ob mice compared with controls [25], suggesting that it's increased expression may enhance fatty acid transport.

The adipokine lipocalin 2 (LCN2) is a member of the lipocalin family that transports small lipophilic ligands and is highly expressed by fat cells both in vitro and in vivo [26]. Levels of LCN2 mRNA are dramatically increased in the adipose tissue and liver of ob/ob mice, while reducing $L C N 2$ expression leads to a decrease in PPARY expression [27]. However, we found that $L C N 2$ was down-regulated in the $\mathrm{BH}$ group compared with the $\mathrm{BL}$ group of pigs. Perilipin 2 (PLIN2) is a cytosolic protein that promotes the formation and stabilization of intracellular lipid droplets, which are organelles involved in the storage of lipid 
depots. PLIN2 polymorphisms have been associated with carcass traits including backfat thickness in pigs [28], while increased PLIN2 mRNA expression was detected in the skeletal muscle of pigs with higher intermuscular fat $[28,29]$. Our results contradict this by revealing a lower PLIN2 mRNA expression level in the livers of pigs with higher backfat thickness. Finally, CYP1A1, CYP1A2, $C Y P 2 A 6$, and CYP26A1 belong to the highly diverse CYP450 super family, and also showed a differential expression pattern in the current study. CYPs have been shown to play critical roles in catalyzing metabolism reactions and in the oxidation of unsaturated fatty acids [30]. Because several differences appear to exist in observed expression levels between studies, further investigation into expression differences of these genes is warranted to elucidate their role in fat deposition.

The present study identified more pathways that were up-regulated than down-regulated in the fatter group of pigs. Most of these pathways are related to the metabolism of substances such as xenobiotics, drugs, retinol, and tryptophan. The retinoid metabolism pathway has previously been shown to be up-regulated in the liver. Retinoid is crucial for most forms of life, and many studies have identified an association between retinoid and lipid metabolism [31]. Retinoids regulate metabolism by activating specific nuclear receptors, including the retinoic acid receptor and the retinoid $\mathrm{X}$ receptor, an obligate heterodimeric partner for other nuclear receptors including PPARs. This helps to coordinate energy balance [32] and suggests that the liver has a larger burden in fatter compared with leaner pigs.

STRING analysis in the present study indicated that genes regulating metabolism influence those related to heat stress. Heat stress was previously shown to affect lipid peroxidation, causing serious damage to membrane lipids, lipoprotein, and other lipid-containing structures [33]. In pigs, body weight is positively correlated with heat stress [34], but our current results show that mRNA levels of genes related to heat stress are down-regulated in the livers of fatter compared with leaner pigs. Future investigations into the interaction between body weight and heat stress may therefore identify novel methods to show how body weight affects healthy individuals.

\section{Conclusions}

This study undertook transcriptome analysis between two groups of Songliao black pigs with different backfat thicknesses. A total of 92 DEGs were identified between $\mathrm{BH}$ and $\mathrm{BL}$ groups. In concordance with the phenotypic differences, these genes belonged to pathways and gene networks related to lipid metabolism, regulation, and transport. Additionally, the identified DEGs related to heat stress could provide a new method of understanding and combating obesity. Our findings will be of use in understanding liver lipid regulation and in the design of new selection strategies to improve pig production.

\section{Additional files}

Additional file 1: Table S1. Gene expression count in four samples.

Additional file 2: Table S2. Primer sequences of six genes related to lipid metabolism for qRT-PCR.

Additional file 3: Table S3. Liver DEGs between $\mathrm{BH}$ and $\mathrm{BL}$ pigs with biological replicates.

Additional file 4: Table S4. Differentially expressed spliced transcripts and primary transcripts.

\section{Abbreviations}

BH: Group with higher backfat thickness; BL: Group with lower backfat thickness; DAVID: Database for annotation, visualization and integrated discovery; DEG: Differentially expressed gene; Full-sib: Full-sibling; GO-BP: Gene ontology biological processes; GO-CC: GO cellular component; GO-MF: GO molecular function; LCN2: Lipocalin 2; PLIN2: Perilipin 2; PPAR: Proliferator-activated receptor; TMM: Trimmed mean of M-values; RT: Reverse transcriptase; STRING: Search tool for the retrieval of interacting genes.

\section{Competing interests}

The authors declare that they have no competing interests.

\section{Authors' contributions}

KX carried out the experiment and drafted the manuscript. ZCH and CDW conceived the study, participated in its design and coordination, and helped draft the manuscript. FZ, HJL and ZJW helped sample, experiment and analysis of data. All authors read and approved the final manuscript.

\section{Acknowledgment}

This research was financially supported by the innovation research team for modern agricultural industry and technology in Beijing.

\section{Author details}

${ }^{1}$ National Engineering Laboratory for Animal Breeding and MOA Key Laboratory of Animal Genetics and Breeding, Department of Animal Genetics and Breeding, China Agricultural University, 100193 Beijing, China. ${ }^{2}$ Tianjin Ninghe Primary Pig Breeding Farm, Ninghe 301500, Tianjin, China.

Received: 30 March 2014 Accepted: 27 May 2014

Published: 6 June 2014

\section{References}

1. Organ R: Key in regulating appetite and body weight. ; 2012.

2. Suzuki K, Inomata K, Katoh K, Kadowaki H, Shibata T: Genetic correlations among carcass cross-sectional fat area ratios, production traits, intramuscular fat, and serum leptin concentration in Duroc pigs. J Anim Sci 2009, 87:2209-2215.

3. Fontanesi L, Schiavo G, Galimberti G, Calò DG, Scotti E, Martelli PL, Buttazzoni L, Casadio R, Russo V: A genome wide association study for backfat thickness in Italian Large White pigs highlights new regions affecting fat deposition including neuronal genes. BMC Genomics 2012, 13:583.

4. Houpt KA, Houpt TR, Pond WG: The pig as a model for the study of obesity and of control of food intake: a review. Yale J Biol Med 1979, 52:307.

5. Reiter SS, Halsey CH, Stronach BM, Bartosh JL, Owsley WF, Bergen WG: Lipid metabolism related gene-expression profiling in liver, skeletal muscle and adipose tissue in crossbred Duroc and Pietrain pigs. Comp Biochem Physiol Part D Genomics Proteomics 2007, 2:200-206.

6. Fam BC, Joannides CN, Andrikopoulos S: The liver: Key in regulating appetite and body weight. Adipocyte 2012, 1:259-264.

7. Muñoz R, Estany J, Tor M, Doran O: Hepatic lipogenic enzyme expression in pigs is affected by selection for decreased backfat thickness at constant intramuscular fat content. Meat Sci 2013, 93:746-751.

8. Nguyen P, Leray V, Diez M, Serisier S, Bloc'h JL, Siliart B, Dumon H: Liver lipid metabolism. J Anim Physiol Anim Nutr 2008, 92:272-283. 
9. Chen C, Ai H, Ren J, Li W, Li P, Qiao R, Ouyang J, Yang M, Ma J, Huang L: A global view of porcine transcriptome in three tissues from a full-sib pair with extreme phenotypes in growth and fat deposition by paired-end RNA sequencing. BMC Genomics 2011, 12:448.

10. Ramayo-Caldas Y, Mach N, Esteve-Codina A, Corominas J, Castelló A, Ballester M, Estellé J, Ibáñez-Escriche N, Fernández Al, Pérez-Enciso M: Liver transcriptome profile in pigs with extreme phenotypes of intramuscular fatty acid composition. BMC Genomics 2012, 13:547.

11. Gunawan A, Sahadevan S, Cinar MU, Neuhoff C, Große-Brinkhaus C, Frieden L, Tesfaye D, Tholen E, Looft C, Wondim DS: Identification of the Novel Candidate Genes and Variants in Boar Liver Tissues with Divergent Skatole Levels Using RNA Deep Sequencing. PLoS One 2013, 8:e72298.

12. Trapnell C, Roberts A, Goff L, Pertea G, Kim D, Kelley DR, Pimentel H, Salzberg SL, Rinn JL, Pachter L: Differential gene and transcript expression analysis of RNA-seq experiments with TopHat and Cufflinks. Nat Protoc 2012, 7:562-578.

13. Quinlan AR, Hall IM: BEDTools: a flexible suite of utilities for comparing genomic features. Bioinformatics 2010, 26:841-842.

14. Delhomme N, Padioleau I, Furlong EE, Steinmetz LM: easyRNASeq: a bioconductor package for processing RNA-Seq data. Bioinformatics 2012, 28:2532-2533.

15. Dillies MA, Rau A, Aubert J, Hennequet-Antier C, Jeanmougin M, Servant N, Keime C, Marot G, Castel D, Estelle J, Guernec G, Jagla B, Jouneau L, Laloë D, Le Gall C, Brigitte Schaëffer B, Le Crom S, Guedj MM, Jaffrézic F: A comprehensive evaluation of normalization methods for Illumina high-throughput RNA sequencing data analysis. Brief Bioinform 2013, 14:671-683.

16. Tarazona S, García-Alcalde F, Dopazo J, Ferrer A, Conesa A: Differential expression in RNA-seq: a matter of depth. Genome Res 2011, 21:2213-2223.

17. Tarazona S, Furió-Tarı P, Ferrer A, Conesa A: NOISeq: Differential Expression in RNA-seq; 2013

18. Huang DW, Sherman BT, Lempicki RA: Systematic and integrative analysis of large gene lists using DAVID bioinformatics resources. Nat Protoc 2009, 4:44-57.

19. Franceschini A, Szklarczyk D, Frankild S, Kuhn M, Simonovic M, Roth A, Lin J, Minguez $P$, Bork $P$, von Mering C: STRING v9. 1: protein-protein interaction networks, with increased coverage and integration. Nucleic Acids Res 2013, 41:D808-D815.

20. Wu J, Zhou D, Deng C, Xiong Y, Lei M, Li F, Jiang S, Zuo B, Zheng R: Expression pattern and polymorphism of three microsatellite markers in the porcine CA3 gene. Genet Sel Evol 2008, 40:227-239.

21. Ousova O, Guyonnet-Duperat $V$, lannuccelli N, Bidanel JP, Milan D, Genet $C$, Llamas B, Yerle M, Gellin J, Chardon P, Emptoz-Bonneton A, Pugeat M, Mormède $P$, Moisan $\mathrm{M}$ : Corticosteroid binding globulin: a new target for cortisol-driven obesity. Mol Endocrinol 2004, 18:1687-1696.

22. Jiang YZ, Li XW, Yang GX: Sequence characterization, tissue-specific expression and polymorphism of the porcine (Sus scrofa) liver-type fatty acid binding protein gene. Yi Chuan Xue Bao 2006, 33:598-606.

23. Wolfrum C, Borrmann CM, Borchers T, Spener F: Fatty acids and hypolipidemic drugs regulate peroxisome proliferator-activated receptors alpha - and gamma-mediated gene expression via liver fatty acid binding protein: a signaling path to the nucleus. Proc Natl Acad Sci U S A 2001, 98:2323-2328

24. Zhao S, Ren L, Chen L, Zhang X, Cheng M, Li W, Zhang Y, Gao S: Differential expression of lipid metabolism related genes in porcine muscle tissue leading to different intramuscular fat deposition. Lipids 2009, 44:1029-1037.

25. Chen R, Cao Y, Ma X, Wang Y, Zhang H, Zhang W, Zhou D: Characterization of lipid metabolism-related genes in mouse fatty liver; 2012.

26. Yan Q-W, Yang Q, Mody N, Graham TE, Hsu C-H, Xu Z, Houstis NE, Kahn BB, Rosen ED: The adipokine lipocalin 2 is regulated by obesity and promotes insulin resistance. Diabetes 2007, 56:2533-2540.

27. Zhang J, Wu Y, Zhang Y, LeRoith D, Bernlohr DA, Chen X: The role of lipocalin 2 in the regulation of inflammation in adipocytes and macrophages. Mol Endocrinol 2008, 22:1416-1426.

28. Davoli R, Gandolfi G, Braglia S, Comella M, Zambonelli P, Buttazzoni L, Russo V: New SNP of the porcine Perilipin 2 (PLIN2) gene, association with carcass traits and expression analysis in skeletal muscle. $\mathrm{Mol} \mathrm{Biol}$ Rep 2011, 38:1575-1583.

29. Gandolfi G, Mazzoni M, Zambonelli P, Lalatta-Costerbosa G, Tronca A, Russo V, Davoli R: Perilipin 1 and perilipin 2 protein localization and gene expression study in skeletal muscles of European cross-breed pigs with different intramuscular fat contents. Meat Sci 2011, 88:631-637.

30. Lewis DF: 57 varieties: the human cytochromes P450. Pharmacogenomics 2004, 5:305-318.

31. Keller H, Dreyer C, Medin J, Mahfoudi A, Ozato K, Wahli W: Fatty acids and retinoids control lipid metabolism through activation of peroxisome proliferator-activated receptor-retinoid $X$ receptor heterodimers. Proc Natl Acad Sci U S A 1993, 90:2160-2164.

32. Ziouzenkova O, Plutzky J: Retinoid metabolism and nuclear receptor responses: New insights into coordinated regulation of the PPAR-RXR complex. FEBS Lett 2008, 582:32-38.

33. Altan Ö, Pabuçcuoğlu A, Altan A, Konyalioğlu S, Bayraktar H: Effect of heat stress on oxidative stress, lipid peroxidation and some stress parameters in broilers. Br Poultry Sci 2003, 44:545-550

34. Ingram $D$, Legge K: Effects of environmental temperature on food intake in growing pigs. Comp Biochem Physiol A Physiol 1974, 48:573-581.

doi:10.1186/2049-1891-5-32

Cite this article as: Xing et al:: The liver transcriptome of two full-sibling Songliao black pigs with extreme differences in backfat thickness. Journal of Animal Science and Biotechnology 2014 5:32.

\section{Submit your next manuscript to BioMed Central and take full advantage of:}

- Convenient online submission

- Thorough peer review

- No space constraints or color figure charges

- Immediate publication on acceptance

- Inclusion in PubMed, CAS, Scopus and Google Scholar

- Research which is freely available for redistribution 